\title{
Imaging of Encephalic Meningiomas (About 142 Cases)
}

\author{
Hicham.Jalal $^{* *}$, B.Bannar ${ }^{1}$, M.Moulattaf ${ }^{1}$, Z.Benyaich ${ }^{2}$, F. El hajjhouji ${ }^{2}$, K.Aniba ${ }^{2}$, H.Ghanane ${ }^{2}$, S. Ait ben ali $^{2}$
}

\author{
${ }^{1}$ Department of Radiology Mother and Child Hospital CHU Mohamed VI Marrakech Morocco \\ ${ }^{2}$ Department of Neurosurgery Arrazi Hospital and Ibn Tofail Hospital, CHU Mohamed VI Marrakech Morocco
}

DOI: $\underline{10.36347 / \text { sjmcr.2021.v09i04.020 }}$

| Received: 06.03.2021 | Accepted: 25.03.2021 | Published: 22.04.2021

*Corresponding author: Hicham.Jalal

Abstract

Original Research Article

Objective: To analyze the contribution of cross-sectional imaging (CT and MRI) in the etiologic and topographic diagnosis of encephalic meningiomas. Material and methods: Retrospective study of 142 cases of encephalic meningiomas collected over an 11 year period between January 2004 and November 2016. 73 patients underwent CT scans without and with iodinated contrast injection, and brain MRI was performed in 69 patients in sagittal T1, axial $\mathrm{T} 2$, and flair sequences and in multiplanar sequences after gadolinium injection. Results: The average age of our patients was 45.10 years with extremes ranging from 12 to 80 years, with a clear female predominance $(75 \%)$. The meningiomas were located in the convexity in $37 \%$ of cases, at the base of the skull in $21 \%$ of cases, in the false brain and para sagittal in $10 \%$ of cases. $60 \%$ of the meningiomas were of meningothelial type. Conclusion: Meningiomas are benign tumors developed from arachnoid cells. They represent $15 \%$ of intracranial tumors, and are supratentorial in $90 \%$ of cases. Through our series and a review of the literature, we analyze the interest of CT and MRI in the topographic diagnosis, lesion assessment and follow-up of meningiomas.

Keywords: Meningiomas, imaging, CT, MRI, histology.

Copyright $\odot 2021$ The Author(s): This is an open-access article distributed under the terms of the Creative Commons Attribution 4.0 International License (CC BY-NC 4.0) which permits unrestricted use, distribution, and reproduction in any medium for non-commercial use provided the original author and source are credited.

\section{INTRODUCTION}

Intracranial meningiomas are extra parenchymal tumors usually of benign histology developing from arachnoid cells of the meningeal envelope. They represent between 24 and $30 \%$ of all intracranial tumors and are supra tentorial in $90 \%$ of cases [3]. They mainly affect adults with a peak frequency between 60 and 70 years of age and a clear female predominance $[6,7]$. Clinical symptoms are often delayed and of imprecise localization value due to the location and volume of the tumor and the nerve or vascular structures that it compresses, underlining the predominant role of imaging based on CT and MRI in their diagnosis. The objective of this article is to analyze the contribution of cross-sectional imaging (CT and MRI) in the positive diagnosis, lesion assessment and topography of these tumors.

\section{Materials ANd Methods}

This is a retrospective study of a series of 142 cases of intracranial meningiomas collected in the radiology and neurosurgery departments of the Mohamed VI University Hospital of Marrakech over a period of 11 years (from January 2004 to November 2016). 73 patients underwent a cerebral CT examination performed by a helical scanner, in axial slices, sometimes associated with coronal slices, of $5 \mathrm{~mm}$ thickness without and with iodinated contrast injection. Brain MRI was performed on a 1.5 Tesla machine in 69 patients, in T1-weighted axial, T2-weighted sagittal, and T2 Flair-weighted axial sequences, supplemented by gadolinium injection in all three spatial planes. Diagnostic confirmation was obtained on anatomopathologic data in all cases.

\section{RESULTS}

The mean age of our patients was 45.10 years with extremes ranging from 12 to 80 years with a great preponderance of tumor occurrence in the 4th decade estimated at $38 \%$. The female sex was predominant, representing $75 \%$ of the cases. The clinical symptomatology was variable, represented essentially by an HTIC syndrome in $42 \%$ of cases, followed by epileptic seizures in $26 \%$ of cases and a motor deficit in $18 \%$ of cases. Visual disturbances were found in $14 \%$ of cases, in the form of decreased visual acuity in 18 patients, visual field disturbances in 11 cases and blindness observed in 8 patients.

The histological type was specified in all our patients, based on the WHO 2000 histological classification. The results are summarized in Table 1 . 
Table-1: Distribution of the cases according to the histological type

\begin{tabular}{|l|l|l|l|}
\hline histologique Grade & Type histologique & Number & $\mathbf{\%}$ \\
\hline Grade I, Classique 85\% & Meningothelial & 85 & 60 \\
\cline { 2 - 4 } & Psammomatous & 14 & 10 \\
\cline { 2 - 4 } & Fibroblastic & 7 & 5 \\
\cline { 2 - 4 } & cystic & 6 & 4 \\
\cline { 2 - 4 } & Transitional & 4 & 3 \\
\cline { 2 - 4 } & Angiomatous & 3 & 2 \\
\hline \multirow{4}{*}{ Grade II, Atypical 11\% } & Anaplasitic & 13 & 9 \\
\cline { 2 - 4 } & Clear Cell & 4 & 3 \\
\hline Grade III, Malignant $\mathbf{4 \%}$ & Malignant & 6 & 4 \\
\hline
\end{tabular}

Topographically, the meningiomas affected the convexity in $37 \%$ of cases and were located at the base of the skull in $21 \%$ of cases. The different sites of the meningiomas in our series are grouped in Table 2.

Table-2: Topography of meningiomas

\begin{tabular}{|l|l|l|}
\hline Locations & Number of cases & Percentage \\
\hline convexity & 52 & 37 \\
\hline Base of the skull & 30 & 21 \\
\hline Sphénoïdal rigide and cavernous sinus & 26 & 18 \\
\hline parasagittal and brain scythe & 22 & 15 \\
\hline intra et suprasellaire & 9 & 7 \\
\hline FCP & 3 & 2 \\
\hline Intraventricular & $\mathbf{0}$ & $\mathbf{0}$ \\
\hline
\end{tabular}

The diagnosis of meningioma was made by brain $\mathrm{CT}$ in all cases due to the suggestive scannographic findings. The tumour was spontaneously iso-dense in $48 \%$ of cases and intensely and homogeneously enhancing in $52 \%$ of cases. It was responsible for adjacent bone changes in $38 \%$ of cases, such as osteocondensation in 18 cases and osteolysis in 2 cases. It was accompanied by peri-lesional oedema in $67 \%$ of cases and exerted a mass effect on the neighbouring structures in $76 \%$ of cases. The different scannographic signs of meningiomas in our series are summarised in Table 3.

Table-3: CT aspects of meningiomas

\begin{tabular}{|l|l|l|}
\hline & Number & $\%$ \\
\hline Spontaneous contrast & & \\
Isodense & 36 & 49 \\
Hyperdense & 32 & 44 \\
hypodense & 5 & 7 \\
\hline enhancement & & \\
Intense et homogenous & 36 & 50 \\
Intense et heterogenous & 29 & 40 \\
Moderate and heterogenous & 4 & 5 \\
\hline Scattered calcifications & 29 & 41 \\
Fully calcified & 9 & 13 \\
\hline Cystic component & 5 & 7 \\
\hline Necrotic component & 13 & 18 \\
\hline Periwound oedema & 59 & 81 \\
\hline Mass effect & 53 & 73 \\
\hline Active hydrocephalus & 45 & 62 \\
\hline Bone condensation & 31 & 43 \\
\hline Bone lysis & 5 & 7 \\
\hline
\end{tabular}

The CT scan was also used to analyse the accompanying signs of the tumour process, notably

- Subfalcoral involvement in 38 cases and temporal involvement in 7 cases

- Infiltration of the superior longitudinal sinus in 3 cases
- Infiltration of the optochiasmatic cistern and repression of the optic chiasm in 5 cases

- Orbital extension en 9 cases.

Brain MRI, performed in 69 patients, was also diagnostic in all cases. The meningioma was isointense in the white matter in $\mathrm{T} 1$ in $88 \%$ of cases, hyperintense 
in $\mathrm{T} 2$ in $56 \%$ and intensely and homogeneously gadolinium-enhanced in $72 \%$ of cases. Thickening with meningeal enhancement adjacent to the tumour was seen in $53 \%$ of cases. MRI revealed perilesional oedema in $41 \%$ of cases and a mass effect in $68 \%$ of cases. The different MRI aspects of the meningiomas in our series are grouped in table $\mathrm{N}^{\circ} 4$.

Table-4: MRI aspects of meningiomas

\begin{tabular}{|l|l|l|}
\hline \multicolumn{2}{|c|}{ Table-4: MRI aspects of meningiomas } & \% \\
T1 & Patients & $\mathbf{7 5}$ \\
Iso signal homogenous & $\mathbf{5 2}$ & $\mathbf{1 3}$ \\
Iso signal heterogeneous & $\mathbf{9}$ & $\mathbf{6}$ \\
Hyposignal & $\mathbf{5}$ & $\mathbf{6}$ \\
hypersignal & $\mathbf{5}$ & \\
\hline T2 & & 15 \\
Isosignal homogeneous & 10 & 26 \\
Isosignal heterogenous & 17 & 53 \\
Hypersignal homogeneous & 36 & 4 \\
Hypersignal heterogeneous & 3 & 2 \\
Hyposignal & 2 & \\
\hline Raising & & 72 \\
Intense homogeneous & 49 & 28 \\
Intense heterogeneous & 19 & 53 \\
\hline Meningeal and enhancement & 36 & 41 \\
thickening & & 68 \\
\hline Perilesional oedema & 28 & 13 \\
\hline Masse effect & 46 & \\
\hline Calcifications & 9 & \\
\hline
\end{tabular}

MRI was also used to analyse the accompanying signs of the tumour process, including

- Trapping of the CSF in 35 cases.

- Vascular structures were blocked in 27 cases.

- Invasion of the superior longitudinal sinus in 19 cases.

- Infiltration of the cavernous sinus in 17 cases.
- Active hydrocephalus in 14 cases.

- Subfalcoral involvement in 15 cases.

- Infiltration of the optic chiasma and filling of the optochiasmatic cisterns in 13 cases.

- Endo-orbital extension in 5 cases.

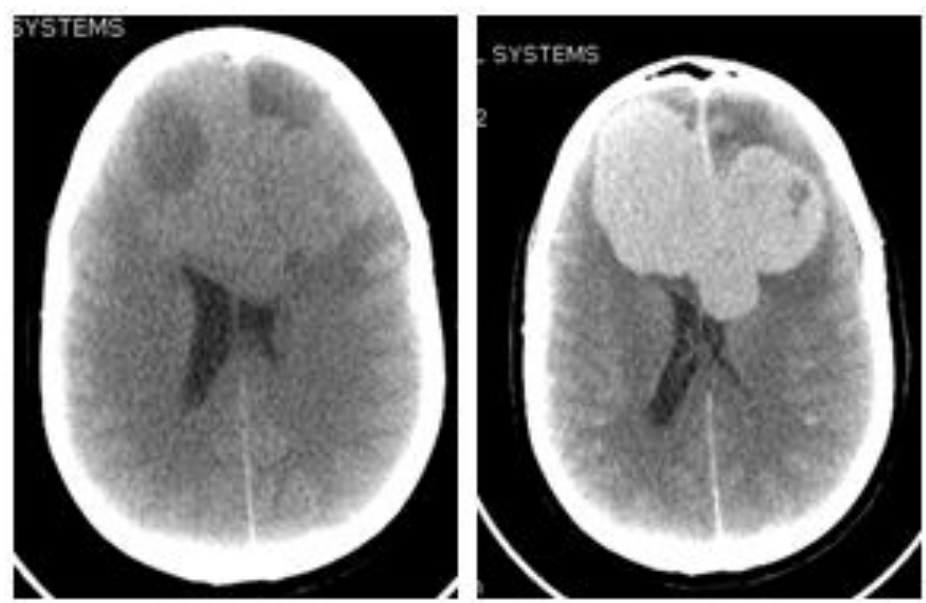

Fig-1: Cerebral CT scan before and after injection of contrast medium: large bi-frontal meningioma of the scythe of the brain, discretely hyperdense in spontaneous contrast, intensely enhancing after injection of contrast medium, surrounded by a discrete edema and exerting a mass effect on the frontal horns 


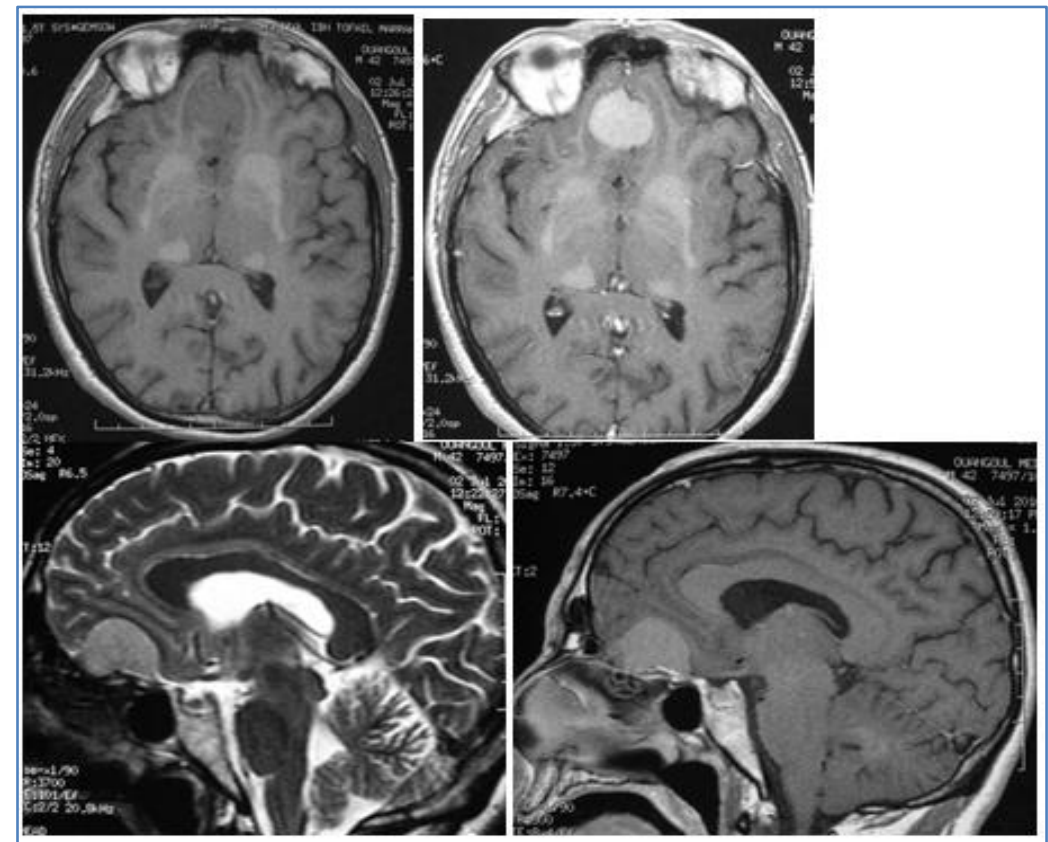

Fig-2: Brain MRI. 42-year-old female patient with a meningioma of the sphenoidal jugum + Sd de far (hypersignal of basal ganglia)

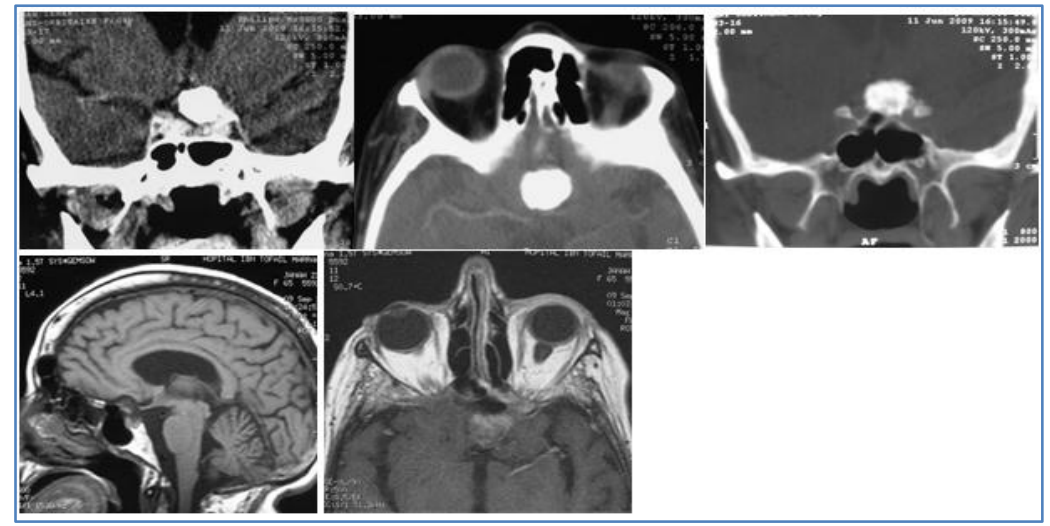

Fig-3: Diabetic patient, 65 years old, visual acuity decrease since 2 months: Calcified suprasellar tumor process infiltrating the chiasma and the optic nerve: psammomatous meningioma of the sphenoidal jugum.
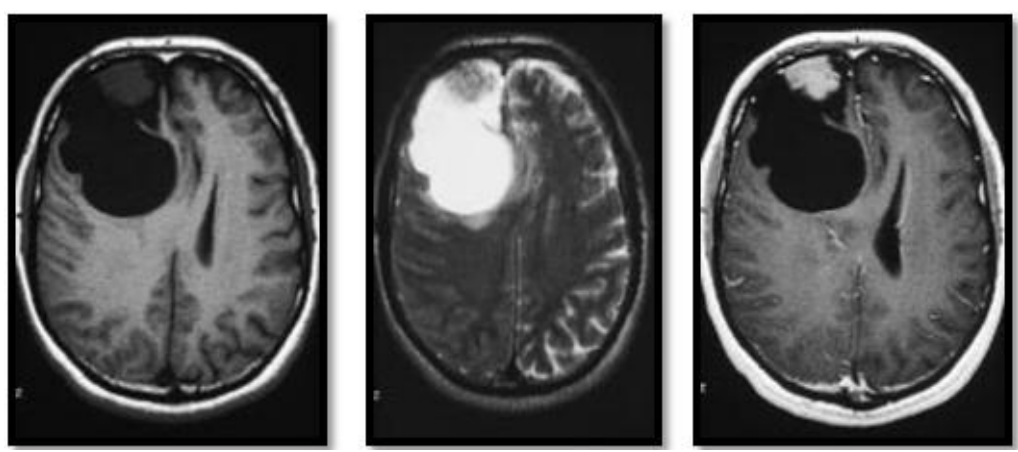

Fig-4: Brain MRI: right frontal cystic meningioma. Right frontal expansive process, with two components, peripheral fleshy isointense to grey matter in $\mathrm{T} 1$ and $\mathrm{T} 2$ and strongly enhancing after gadolinium injection and cystic, very hypointense $\mathrm{T} 1$, very hyperintense T2, not enhancing after gadolinium, with polylobed and compartmentalized contours 

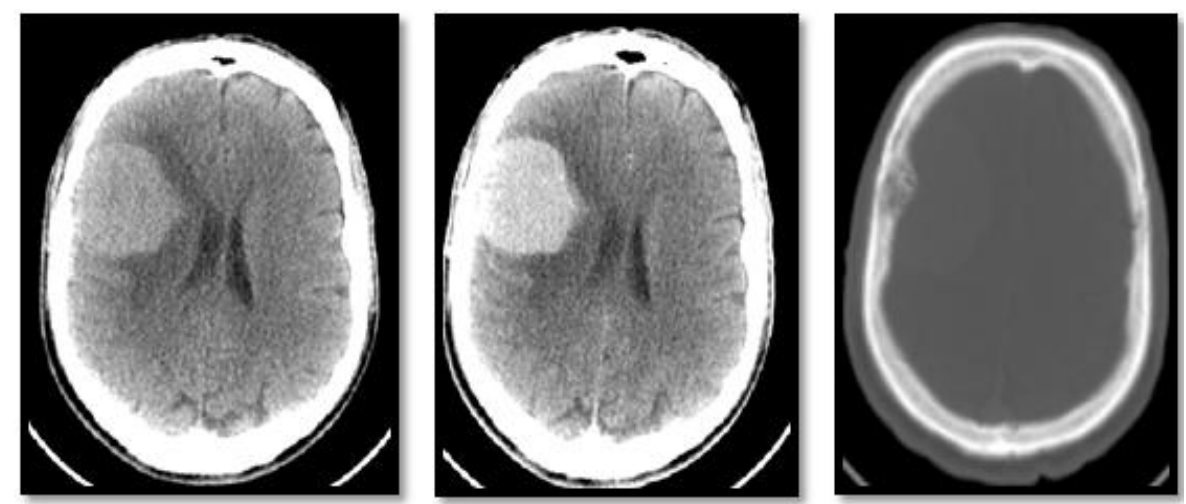

Fig-5: Brain CT: right convexity meningioma: tumor process, well limited, spontaneously hyperdense, intensely and homogeneously enhancing, with a large meningeal implantation base, surrounded by a discrete peri-lesional edema, exerts a mass effect on the VL and is accompanied by an opposite osteocondensation
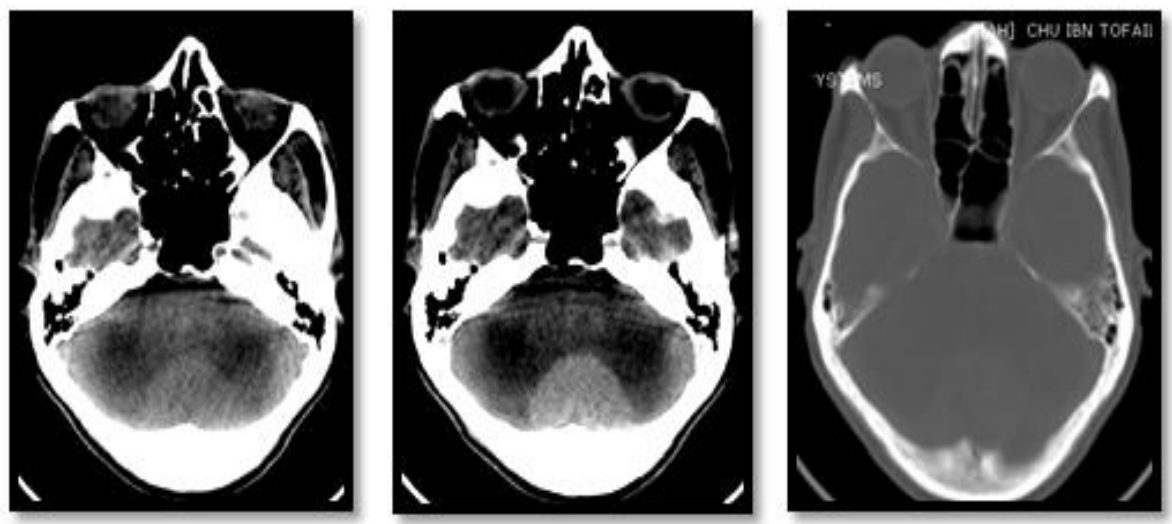

Fig-6: brain CT before and after injection of contrast medium: meningioma of the cerebellum tent: discreetly hyperdense in spontaneous contrast, intensely and homogeneously enhanced, surrounded by a peri-lesional edema and compressing the V4 with upstream hydrocephalus
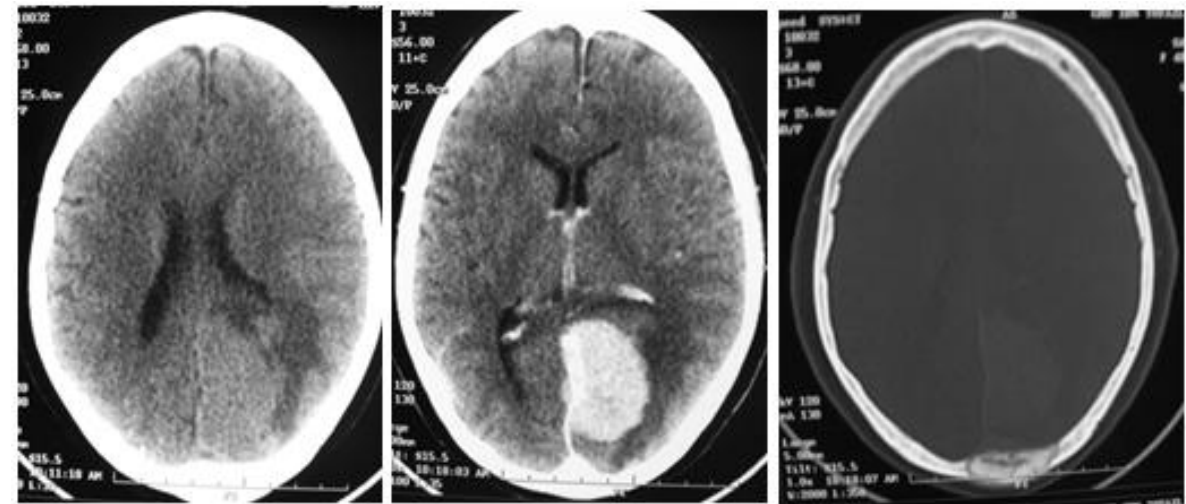

Fig-7: Cerebral CT in axial sections without and with contrast injection. Left occipital meningioma responsible for an opposite hyperostosis
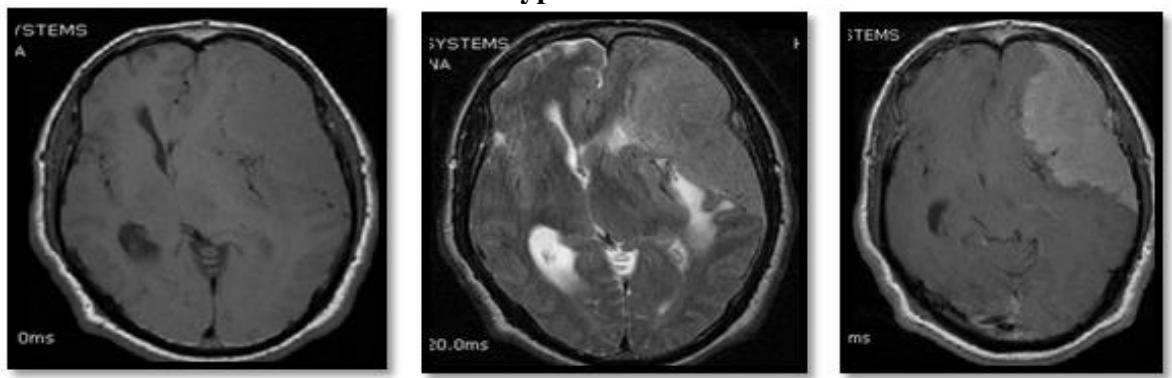

Fig-8: Brain MRI: meningioma of the left convexity. T1: left fronto-parietal tumor process in T1 isosignal, wide meningeal implantation base, exerting a mass effect on the midline + subfalcoral involvement. T2: homogeneous hypersignal, perilesional edema. $T 1+C$ : intense and homogeneous contrast 

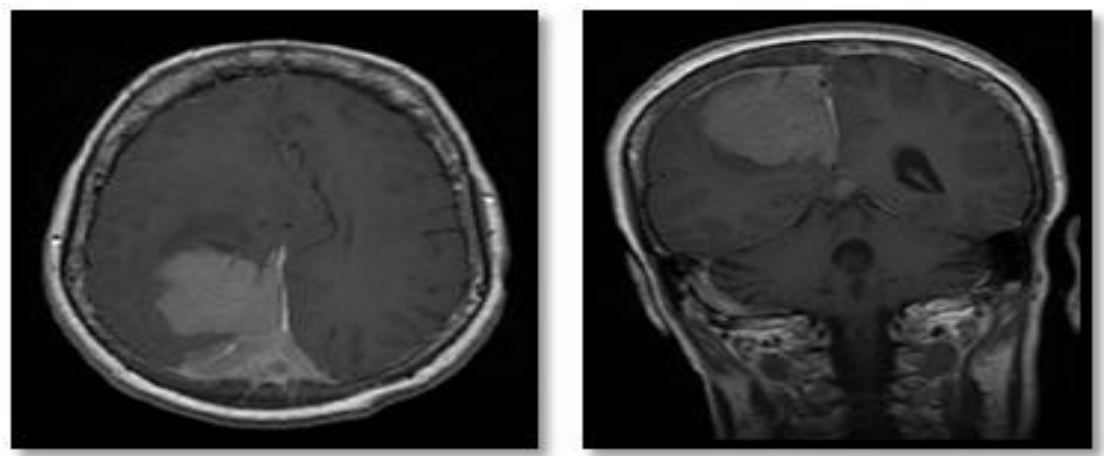

Fig-9: Brain MRI: Right posterior parietal meningioma, homogeneously enhancing after gadolinium injection, associated with meningeal thickening and contrast and invasion of the superior longitudinal sinus
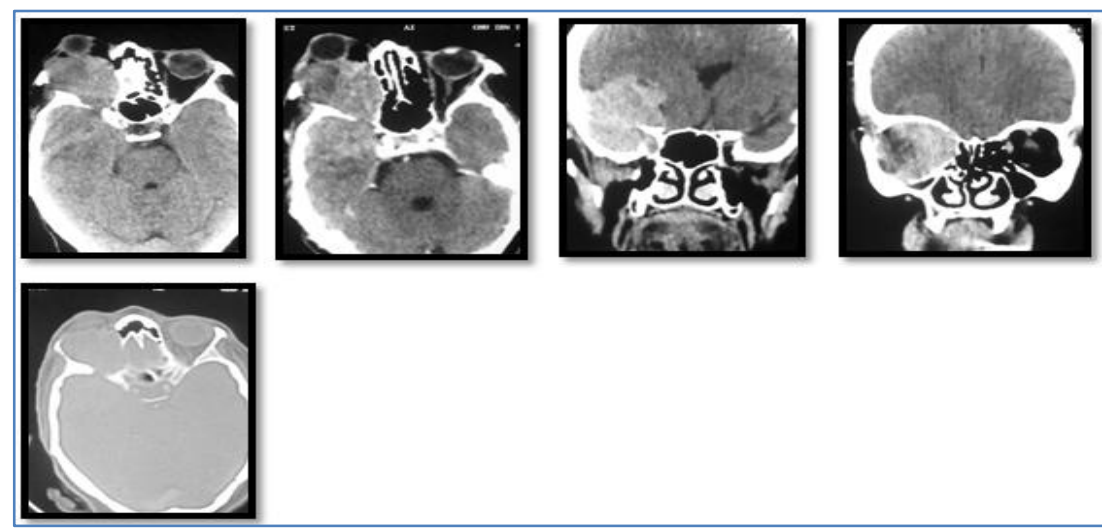

Fig-10: Brain CT: right temporal meningioma with endo-orbital extension. Spontaneously isodense right temporal tumor process, intensely and heterogeneously enhancing; presents a large meningeal implantation base at the level of the greater sphenoid wing with osteocondensation opposite. It extends into the endo-orbital region, engages the optic nerve and infiltrates the oculomotor muscles with grade III exophthalmos
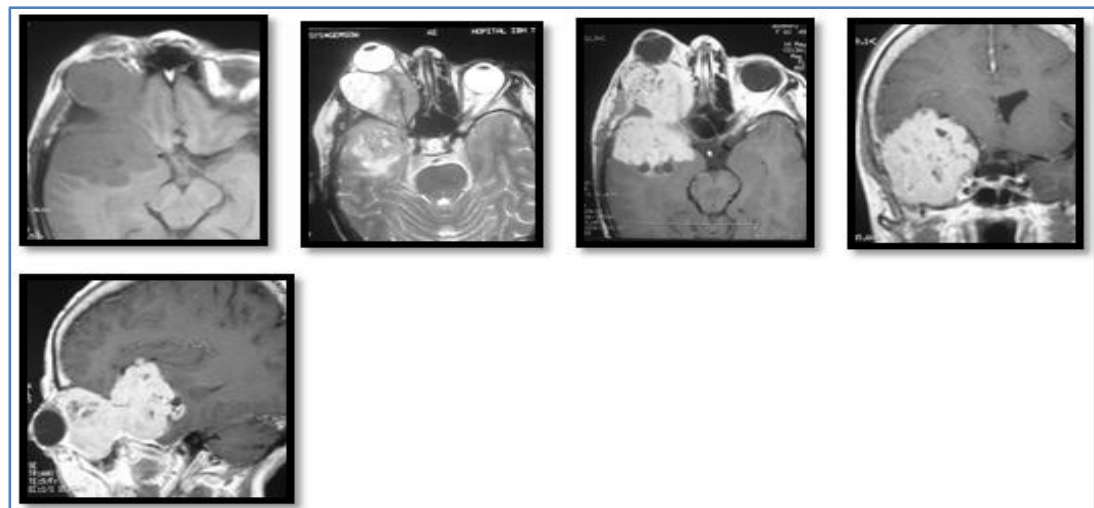

Fig-11: Brain MRI of the same patient: spheno-temporo-orbital meningioma: right temporal tumor process, hyposignal T1, heterogeneous hypersignal $\mathrm{T} 2$, intense and heterogeneous contrast. The lesion is separated from the brain parenchyma by CSF. It exerts a mass effect on the frontal and temporal horns, the optochiasmatic cistern and the cerebral pedicle. It extends through the sphenoidal cleft into the endo-orbital region with stage III exophthalmos 


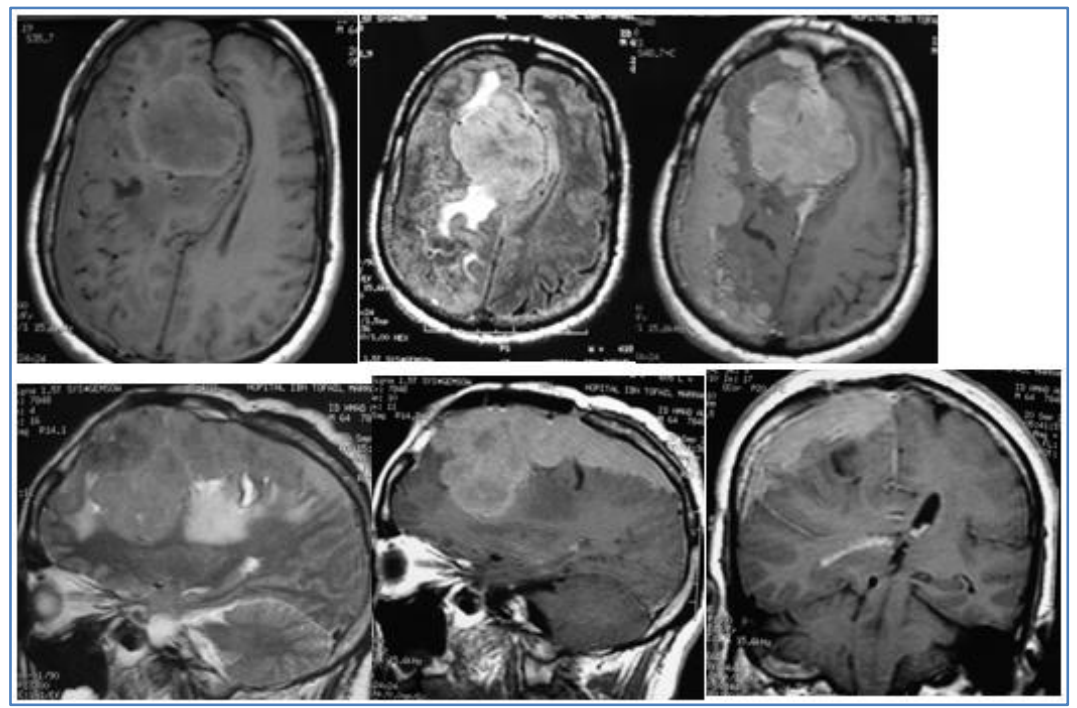

Fig-12: Brain MRI: Operated right frontal meningioma, recurrence with invasion of the bone opposite. Voluminous frontotemporo-parietal tumor recurrence with involvement under the brain scythe and through the cranial flap with invasion of the superior longitudinal sinus

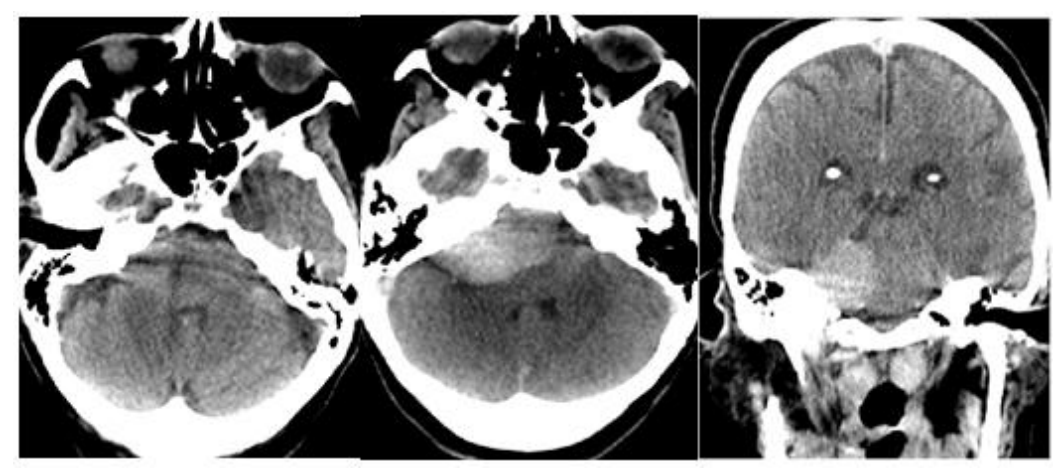

Fig-13: Brain CT: Meningioma of the right cerebellopontine angle. -C: spontaneously isodense APC tumor process exerting a mass effect on the brainstem and V4. $+\mathrm{C}$ in axial and coronal sections: intense and homogeneous contrast with a wide meningeal implantation base
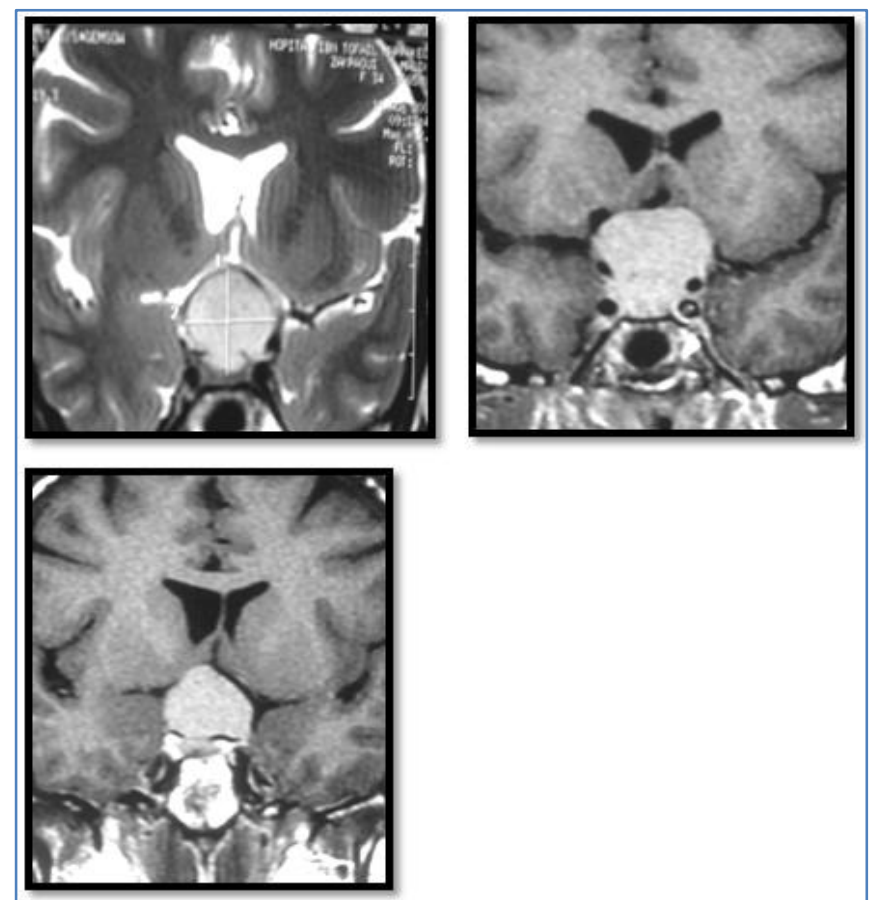

Fig-14: Intra- and suprasellar meningioma: intra- and suprasellar tumor process in homogeneous T2 hypersignal, intensely and homogeneously enhancing. It infiltrates the optic chiasma and engulfs the cavernous sinuses 

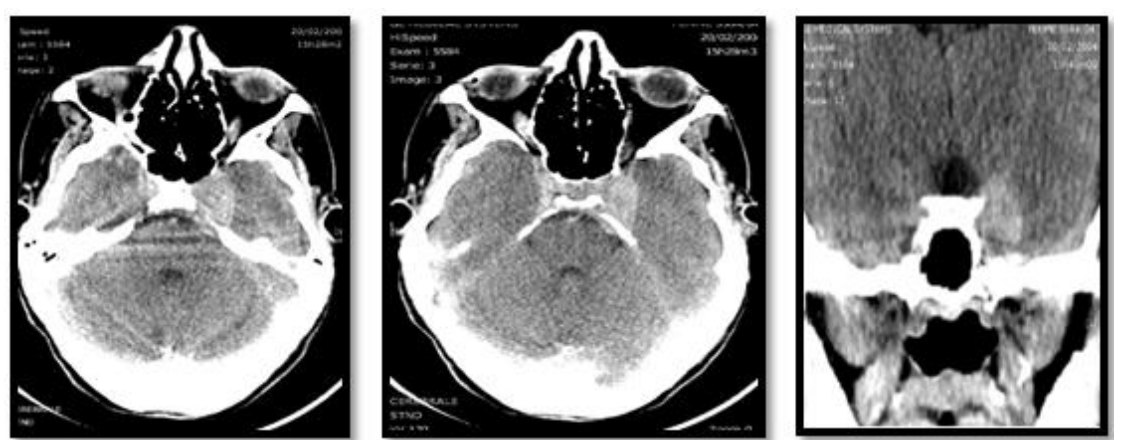

Fig-15: Cerebral CT in axial and coronal sections without and with PDC injection: meningioma of the left cavernous sinus

\section{DISCUSSION}

Meningioma is an extra-axial, benign, well encapsulated tumour originating from the cells of the arachnoid villi which invade the veins and the dura mater sinuses in a "finger of glove" fashion. This tumour represents $15-20 \%$ of primary intracranial tumours and ranks third after metastases and gliomas [1]. The predominance of females from the age of 45 onwards is an overall characteristic of meningiomas, confirmed by several studies, whereas a male predominance is noted for aggressive meningiomas. Children and adolescents are exceptionally affected. Among the predisposing factors, it is classic to retain a type II neurofibromatosis affecting the young subject with multiple meningiomatous localisations; thus the discovery of a meningioma before the age of 10 years should systematically lead to a search for a type II neurofibromatosis [2]. Other factors are represented by radiotherapy which induces an alteration of chromosome 22, hormonal modifications, in particular progesterone, or cranial trauma. All topographies are possible for meningiomas with some preferential locations, with, in decreasing order of frequency, convexity meningiomas (30\%), para-sagittal and scythe meningiomas (30\%) (fig.1), jugo-olfactory meningiomas (15\%) (fig.2), meningiomas of the sphenoidal ridge and cavernous sinuses (13\%), meningiomas of the posterior cerebral fossa (PCF) (9\%); multiple meningiomas outside neurofibromatosis type II (5\%); Meningiomas of the optic nerve sheath and intraventricular nerves are rare; ectopic meningiomas are exceptional and may be located in the diploea, orbit, nasal cavities and nasopharynx [2].

The WHO classification recognises three types of increasing evolutivity:

- Grade I meningiomas: benign meningiomas (meningothelial, fibroblastic, transitional, psammomatous, microcystic, angiomatous and metaplastic types).

- Grade II meningiomas: with a high risk of recurrence or aggressive behaviour (atypical, clear cell and chordoid meningiomas).

- Grade III meningiomas: malignant meningiomas (rhabdoid, papillary and anaplastic meningiomas).

Many meningiomas are asymptomatic and discovered incidentally on CT or MRI. Convexity meningiomas are usually revealed by partial or generalised epileptic seizures in 30-40\%. Motor deficit or visual disturbances are frequently found [1].

Tumours of the PCF mainly involve the cranial nerves, most often the $\mathrm{V}$ nerve with facial hypoesthesia or the VIII nerve with vertigo accompanied by headache without specificity (fig. 6 and 13).

Meningiomas of the sellar region may be revealed by signs of HTIC, by frontal signs or more frequently by visual signs including decreased visual acuity, ophthalmoplegia or exophthalmos (fig.14) [1, 3].

Imaging of meningiomas is based on CT and especially MRI, which firstly allow the extra-axial topography of the expansive process to be confirmed, the positive diagnosis to be made and the lesion to be assessed. The extra-axial topography of the tumour is easy to determine if the tumour is small and remains separated from the cerebral cortex by CSF; An open connection angle at the level of the contact zone with the internal table of the cranial vault, thickening and enhancement of the dura mater, changes in the vault or the base of the skull opposite the tumour in the form of hyperostosis, thinning, bone lysis, or the extension of the tumour towards a venous sinus, constitute arguments in favour of the extra-axial topography of a tumour process (Table $\mathrm{N}^{\circ} 5$ ). 
Hicham.Jalal et al., Sch J Med Case Rep, Apr, 2021; 9(4): 373-383

Table-5: Signs to differentiate between an intra-axial and extra-axial lesion

\begin{tabular}{|l|l|l|}
\hline Grey matter & Lésion intra axiale & Lésion extra axiale \\
\hline Cortical furrrows & invaded & $\begin{array}{l}\text { Repressed or respected } \\
\text { Presence of a cortical band between the } \\
\text { tumour and the oedema }\end{array}$ \\
\hline Subarachnoid space & Non visible & Respected \\
\hline Connection to the vault & deletide & $\begin{array}{l}\text { Enlarged } \\
\text { Presence of fluid and/or vessels between } \\
\text { the tumour and the cortex }\end{array}$ \\
\hline bone & Sharp & Obtuse \\
\hline & $\begin{array}{l}\text { Normal } \\
\text { Inner table notch (DINET, } \\
\text { oligodendroglioma) }\end{array}$ & Hyperostosis ou ostéolysis \\
\hline
\end{tabular}

Computed tomography (CT) with injection of contrast medium represents a reliable means of diagnosis of meningiomas, since its sensitivity is close to $100 \%$. In spontaneous contrast, the majority of meningiomas are hyperdense or isodense. The fibroblastic, transitional and psammomatous forms are the most dense (Fig. 3).

Calcifications may be diffuse or peripheral and are present in 10-20\% [2, 3]. Microcystic meningiomas are hypodense as well as lipoblastic forms containing fatty areas. Meningiomas with hemorrhagic content are exceptional. Injection of contrast medium results in intense enhancement, which is usually homogeneous in the absence of calcifications and cystic areas (Fig. 4).

Enhancement is difficult to evaluate for psammomas because of the predominance of calcifications. Microcystic meningiomas have a weak and progressive enhancement. A hypodense perilesional edema is noted in 50 to $70 \%$ of cases, more marked in angioblastic and aggressive forms. CT scans are better than MRI at analyzing adjacent bone changes such as hyperostosis; bone lysis is seen in aggressive and malignant forms (Figs. 5, 6, and 7).

At the level of the vault, extracranial extension is sometimes associated with grass fire images posing the problem of differential diagnosis with an osteosarcoma. A spheno-orbital hyperostosis associated with a small meningeal mass is consistent with a plaque meningioma. Convexity hyperostosis associated with a large meningeal mass with extracranial extension corresponds to a mass meningioma [2] (fig.8). The angioscanner allows a precise analysis of the arterial and venous relationships of the meningioma.

MRI is the reference exploration of meningiomas. It specifies the extra-axial topography of the lesion, evaluates its repercussions on the brain parenchyma and assesses the arterial and venous relationships. In T1-weighted image, meningiomas are isointense in the gray matter in $60 \%$ and discreetly hypointense in $40 \%$. A T1 hyperintense intra-tumor area corresponds to a hemorrhagic or lipid component, whereas T1 hypointense areas correspond to calcifications or cystic and/or necrotic areas [2, 4]. Psammoma is strongly hypointense (Fig. 3). Meningothelial meningioma presents a radial structure (50\%) with trabeculae in hyposignal in $\mathrm{T} 1$ and $\mathrm{T} 2$ converging towards the insertion pedicle. In proton density, T2 and Flair, the meningioma is either isointense to the cerebral cortex $(50 \%)$, or hyperintense $(50 \%)$. Fibroblastic and transitional forms are isointense, whereas syncytial and angioblastic forms are rather hyperintense on T2. Necrosis or cystic foci appear in frank hypersignal, while calcifications remain in hyposignal. After IV injection of gadolinium, enhancement is intense and homogeneous for small tumors, heterogeneous for larger tumors (cystic or necrotic areas) (Fig. 4).

Psammomas and lipoblastic meningiomas are poorly enhanced and show peripheral linear contrast. Table $\mathrm{N}^{\circ} 6$ shows the MRI aspects of the different subtypes of meningiomas.

Table-6: Aspects IRM des différents sous types des méningiomes

\begin{tabular}{|l|l|l|l|l|l|}
\hline Méningioma & T1 & T2 & Gadolinium & oedema & Necrosis or cyst \\
\hline Meningothelial & Iso / hyposignal & Hyper signal & ++ & ++ & ++ \\
\hline Fibroblastic & Iso/ hyposignal & Iso/ hyposignal & + & + & \\
\hline Angioblastic & Iso/ hyposignal & Hyper signal & ++ & ++ & ++ \\
\hline Transitionnal & Iso/ hyposignal & Iso/ hyposignal & + & + & \\
\hline Psammomatous & Hyposignal & Hypo signal & Linear / peripheral & - & - \\
\hline Papillary & Iso/ hyposignal & Hyper signal & ++ & ++ & \\
\hline Anaplasic & Iso/ hyposignal & Hyper signal & ++ & ++ & \\
\hline Malin & Iso/ hyposignal & Hyper signal & ++ & ++ & \\
\hline
\end{tabular}


The adjacent meningeal thickening and enhancement or "dural tail sign" is suggestive of the diagnosis but remains nonspecific (Figs. 9, 10 and 11). This sign can be associated with all lesions (intra- or extra-axial) developing in contact with the meningeal envelope and reflects edema and hyperhemia rather than tumor infiltration [5]. All these lesions are summarized in table $\mathrm{N}^{\circ} 7$.

\section{Table-7: Variety of lesions that can give the "dovetail sign" (dural tail sign) eningioma \\ Schwanoma of the acoustic Glioma \\ Intra parenchymal metastasis Lymphoma \\ Adenoid cystic carcinoma \\ Sarcoidosis \\ Aneurysm}

Vasogenic oedema is noted in $50 \%$ of cases, in the form of a homogeneous, proton density hyperintense area on T2 and Flair.

The interface between the tumour and the brain parenchyma is variable, represented by a thin hypointense line in $\mathrm{T} 1$, hyperintense in $\mathrm{T} 2$ corresponding to $\mathrm{CSF}$, or contrast in the periphery of the meningioma linked to arachnoid felting noted in Flair after injection of gadolinium (fig.8, 10 and 11). Angiography evaluates the invasion of the venous sinuses in the case of parasagittal meningiomas or meningiomas inserted near the transverse sinus. It also looks for backflow of arterial structures in the case of large prefrontal meningiomas [6].

Perfusion and spectroscopy also provide arguments for a meningeal lesion and help to rule out certain differential diagnoses. Meningothelial meningioma has a highly increased cerebral blood volume ratio (rVSC) (value around 10). Spectroscopically, it is characterised by a peak of alanine, choline and glutamine, a low level of creatine and NAA and little lipid [2, 7]. On the contrary, spectroscopy can be mistaken for a spectral profile of a glial tumour; it is always important to look for simple signs in favour of an extra-axial localisation.

\section{Certain signs on MRI suggest possible operative difficulties}

- The presence of subcortical oedema fed or not by the pial arteries.

- Invasion of the venous sinuses and the main cortical veins. On T1 and T2 sequences, we look for an intravascular lesion with the same behaviour as the meningioma (fig.9 and 15). MRA with gadolinium injection is indicated.

- The proximity of the meningioma to the sylvian artery and the cavernous sinus (fig. 5) limits the possibilities of removal, and treatment is then completed by radiosurgery $[10,11]$.

- The risk of recurrence is greater when there is cortical extension and signs of malignancy [4, 9], (fig.12).

The criteria for malignancy of meningiomas on MRI are

- Large tumour with strong hypersignal

- Necrotic tumour centre

- Absence of calcifications

- Irregular tumour contours

- Rapid progression in size

- Diffusion: reduced ADC in the necrotic centre

- Perfusion: rVSC increased

- Spectroscopy: high Cho/Creat ratio, presence of lipids and lactates, glutamine peak

- Absence of CSF border between tumour and cortex

- Significant intra parenchymal oedema

The differential diagnosis of meningioma can be made with haemangiopericytoma which, although rare $(0.4 \%$ of primary CNS tumours), has the same features on CT and MRI as aggressive meningioma. It can also occur with focal arachnoidodal lesions such as primary lesions of mesenchymal origin (fibromatosis, inflammatory myofibroblastic tumour, leiomyoma, rhabdomyosarcoma, chondrosarcoma, hemangioma, angiosarcoma, Kaposi's sarcoma and epithelioid hemangioendothelioma) or melanocytic (melanocytoma and primary meningeal malignant melanoma), and secondary lesions, which are more frequent [2]. Single arachnoidodural metastasis is rare. Perfusion helps to advance the diagnosis by showing an increased rVSC (>1.5) but more significantly than meningioma (rVSC close to 10 for the meningothelial type); the clinical context is often suggestive and the secondary lesion evolves rapidly. Meningeal pseudotumours may also mimic a meningioma [7].

\section{Conclusion}

Intracranial meningiomas occupy an important place among primary brain tumours. The retrospective study of 97 cases of intracranial meningiomas has allowed us to note the essential contribution of imaging in the assessment of this tumour by allowing an aid to positive diagnosis, a particularly informative topographical analysis and a precise lesion assessment. Currently, magnetic resonance imaging is the best way to evaluate the meningioma by studying its topography, morphology and signal. CT scanning also provides arguments in favour of the diagnosis and represents a reliable diagnostic means apart from certain locations at the base of the skull. The imaging data, when compared with the clinical data, often allow a precise diagnostic orientation, but the diagnostic certainty remains histological. 


\section{REFERENCES}

1. Claus EB, Bondy ML, Schildkraut JM, Wiemels JL, Wrensch M, Black PM. Epidemiology of intracranial meningioma. Neurosurgery. 2005;57:1088-95.

2. Dietemann JL. Neuro-imagerie diagnostique Masson : Imagerie médicale Précis. 2007: 258-287.

3. Drevelegas A. Extra axial brain tumors. Eur Radiol. 2005;15: 453-467.

4. Drape JL, Krause D, Tongio J. MRI of aggressive meningiomas. J Neuro radio. 1992; 9:49-62.

5. Takeguchi T, Miki H, Shimizu T, Kikuchi K, Mochizuki T, Ohue S and Ohnishi T. The dural tail of intracranial meningiomas on fluid-attenuated inversion-recovery images. Neuroradiology. 2004; 46:130-135.

6. Nadel L, Braun IF, Muizelaar JP, Laine FJ. Tumoral thrombosis of cerebral venous sinuses: pre operative diagnosis using magnetic resonance phase imaging. Surg Neurol. 1991; 35:189-195.
7. Majos C, Alonso J, Aguilera C, Serrallonga M, Coll S, Acebes Jj, Arus C And Gili J. Utility of proton MR spectroscopy in the diagnosis of radiologically atypical intracranial meningiomas. 2003; 45:129-136.

8. Chen TC, Zee CS, Miller CA and al. Magnetic resonance imaging and pathological correlates of meningiomas. Neurosurgery. 1992; 31:1015-1021; Discussion 1021-1012.

9. Mantle RE, Lach B, Delgado MR, Baeesa S, Belanger G. Predicting the probability of meningioma recurrence based on the quantity of peritumoral oedema on computerized tomography scanning. J Neurosurg. 1999;91:375-383.

10. Mohda A, Gutin PH. Diagnosis and treatment of atypical and anaplastic meningiomas: a review. Neurosurgery. 2005;57:538-50.

11. Dziuk T, Woo S, Butler EB, Thornby J, Grossman R, Dennis WS. Malignant meningioma: an indication for initial aggressive surgery and adjuvant radiotherapy. J Neurooncol. 1998;37:17788. 